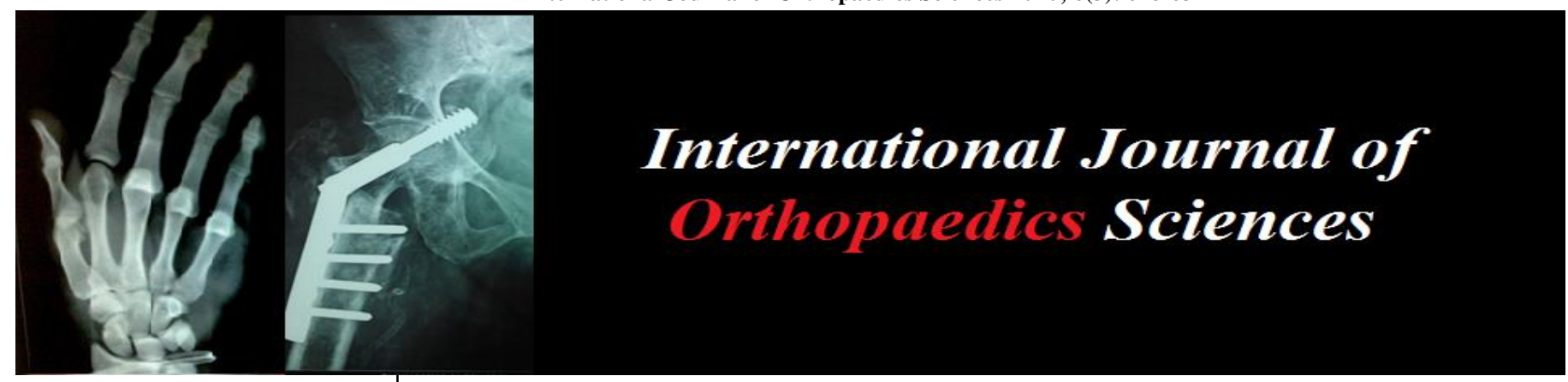

E-ISSN: 2395-1958

P-ISSN: 2706-6630

IJOS 2020; 6(3): 628-632

(C) 2020 IJOS

www.orthopaper.com

Received: 12-05-2020

Accepted: 14-06-2020

\section{Dr. Jatin Sanandia}

Associate Professor Department

of Orthopedics, Ananta Medical

College and Research Centre,

Udaipur, Rajasthan, India

\section{DR. Nitin Kansa}

Consultant Orthopaedic and

Spine Surgeon, Kansal

Hospital, Ghaziabad, Uttar

Pradesh, India

\section{Dr. Rasik Dabhi}

Associate Professor Department of Orthopedics, GMERS

Himmatnagar, Gujarat, India

\section{Dr. Harsh Pate}

Resident Doctor Department of Orthopaedics, SMT NHL MMC, Ahmedabad, Gujarat, India
Corresponding Author: Dr. Harsh Patel

Resident Doctor Department of Orthopaedics, SMT NHL MMC, Ahmedabad, Gujarat, India

\section{A study of operative and non - operative management of dorsolumbar spine fracture (A study OF 40 cases)}

\author{
Dr. Jatin Sanandia, DR. Nitin Kansal, Dr. Rasik Dabhi and Dr. Harsh \\ Patel
}

DOI: https://doi.org/10.22271/ortho.2020.v6.i3j.2262

\section{Abstract}

In an epidemiological study traumatic injury to the thoracolumbar area constitute of $75 \%$ of total back skeletal injuries. Most of these injuries comprises of thoracolumbar segment of the spine (t10 to 12), also high incidence of neurological deficits is associated with thoracolumbar fractures. Numerous classifications were proposed on an anatomical basis to guide the management of TL fractures and define the indications for surgery but none of these classification systems have taken into account the stabilizing effect of para- spinal muscles. The aim of this article is to review the present literature on evidence of management of TL fractures using operative versus conservative techniques. 40 patients with dorsolumbar spine injuries falling into inclusion criteria were managed with conservative and operative intervention between December 2008 to January 2010. 16 patients were treated with operative intervention with short or long segmented fixation \& fusion with bone grafts. 24 patients were treated non operatively with DLSO brace and kept immobilised for 4-6 weeks. The maximum period of follow up was 18 to 24 months with a minimum period of follow up of 9 to 12 months. At follow up on regular check of neurological status, $50 \%$ of operatively treated patients were showing improvement. Patients treated non operatively had no or occasional pain according to pain score. 10 patients had excellent score treated operatively while 12 patients had good score. 8 patients treated non operatively had good to fair score according to grading for neurological improvement by SRS (scoliosis research society). Surgical intervention should be advocated in cases of unstable burst fractures of the dorsolumbar region, irrespective of status of neurological damage, but might be restricted in the patients with incomplete spinal cord injuries.

Keywords: Dorsolumbar spine fractures, operative intervention, non-operative intervention

\section{Introduction}

In an epidemiological study by $\mathrm{Hu}$, et al., traumatic injuries to the thoracolumbar area constitute of $75 \%$ of total back skeletal injuries ${ }^{[1]}$. Most of these injuries comprises of thoracolumbar segment of the spine(t10 to 12), biomechanically the transfer of axially directly kinetic energy from a mobile lumbar spine to a rigid thoracic back causes a high incidence of injuries at this joint ${ }^{[2]}$ it is the most frequent site of injury in spine after cervical spine in adults [3]. A high incidence of neurological deficits is associated with thoracolumbar fractures. Kyphotic deformity, late neurological deterioration, and chronic pain are long-term consequences which can hamper the quality of life ${ }^{[4]}$. Numerous classifications were proposed on an anatomical basis to guide the management of TL fractures and define the indications for surgery but none of these classification systems have taken into account the stabilizing effect of para- spinal muscles. The injured structures are usually capable of natural healing over time and stabilize the fracture site ${ }^{[5]}$

The aim of this article is to review the present literature on evidence of management of TL fractures using operative versus conservative techniques. Two main principles guide the management of TL fractures: biomechanical stability and neurological optimization are the two main principle guide of the management of TL fractures ${ }^{[6]}$

Numerous classification systems have come up to guide the management of TL fractures. Denis classification stressed on the integrity of the middle column ${ }^{[7]}$ Aebi proposed a comprehensive AO classification that combined pathomorphological findings (based on 
imaging) and mechanics of the injury with emphasis on the posterior ligament complex ${ }^{[8]}$. McAfee et al. used computed tomography scan findings for classification ${ }^{[9]}$. The load sharing classification of McCormack et al. proposed a score to determine the approach to surgery. ${ }^{[10]}$ The Thoracolumbar Injury Classification and Severity Score was proposed by Lee et al. to facilitate the clinical decision-making. ${ }^{[1]}$

criteria for instability in a TL burst fracture has still many controversies. Holdsworth defined instability as the rupture of the posterior ligamentous complex. Denis ${ }^{[7]}$ stated that all burst fractures are unstable even when they present without neurodeficits. McAfee [9] pointed out that progressive neurologic deficit, associated posterior element disruption, kyphosis progressing $20^{\circ}$ or more in the presence of a neural deficit, $>50 \%$ loss of vertebral height with facet joint subluxation and CT-demonstrated free bony fragments within a compromised spinal canal associated with an incomplete neural deficit as the indicators of instability. Magerl described the treatment modalities should not be based on the presence/absence of instability but on the type and degree of instability.

There are few arguments regarding favouring of surgical intervention. immediate stabilization of the spine will decrease the chance of neurologic deterioration. surgery will correct kyphosis which will decrease pain and future degenerative change. surgery allows early mobilization, thereby decreasing the complications and costs related to prolonged bed rest. surgical decompression allows the removal of retropulsed fragments from the canal and decreases the chances of neurological deterioration.

Denis ${ }^{[7]}$ reported a neurological deterioration in 6 out of 29 patients with burst fractures but his results were not supported in the subsequent literature. Weinstein et al. ${ }^{[12] ~ h a d ~}$ successfully treated 42 patients conservatively and no case with early or late neurological deterioration was reported. Shen et al. ${ }^{[13]}$ in 2001 speculated that the risk of neurologic injury actually may be higher with surgical management based on his experience. Chow et al. ${ }^{[14]}$ also reported successful conservative management in 26 patients with hyperextension bracing or casting with no neurological deterioration.

Surgical correction stays initially but the studies showed that it much of it will be lost subsequently ${ }^{[15]}$ even though hardware remains intact, kyphosis increases due to mechanisms such as motion at screw plate junction, motion of screw within the bone and fatigue bending. Extension of the fusion may preserve the correction but at the cost of loss of motion segments. [16] McNamara et al. [17] reported a postoperative kyphosis progression of $8.7^{\circ}$ with only $69 \%$ return to routine activity in his 13 surgically treated patients. in the randomized study by Wood et al. ${ }^{[18]}$ there was no significant difference in kyphotic progression between conservative and surgical groups Weinstein et al. ${ }^{[12]}$ stated that kyphosis did not correlate with pain or function at follow up.

The complications of prolonged bed rest can be reduced by early mobilization. Shen and Shen ${ }^{[16]}$ did not find any complications related to early mobilization. mobilization protocols for nonoperative treatment vary from 6 weeks in a roto-rest bed to early mobilization in a brace. bracing is useful for initial pain control. bracing does not prevent the collapse of the vertebrae and probably does not change the long-term result.

Retropulsion of bony fragments into the canal are associated with the burst fractures which can further lead to canal compromise. Canal compromise $>25 \%$ is often quoted as an indication for surgery in fear of neurological deterioration ${ }^{[5]}$. geometry of canal is not an indicator of the extent of neurological dysfunction and therefore surgical decompression does not alter its outcome.

\section{Material and methods}

In a prospective study conducted at our institution, 40 patients with dorsolumbar spine injuries were managed with conservative and operative intervention between December 2008 to January 2010. The maximum period of follow up was 18 to 24 months with a minimum period of follow up of 9 to 12 months.

\section{Inclusion criteria \\ For non-operative patients \\ 1. Stable dorsolumbar vertebral fractures \\ 2. Absent neurological deficit \\ 3. Hemodynamically medically unfit for surgery}

\section{For operative intervention patients}

1. Unstable or fracture dislocations

2. Neurological deficit

\section{Exclusion criteria}

Patients with head, cervical and pelvic injuries and discernible dorsal \& lumbar spine injuries

All patients were treated after stabilising vitally, catheterised for strict input output charting and neurologically assessed thoroughly. Whole head to toe evaluation undertaken for head, chest, abdomen injuries as well as extremity injuries by CT scan, whole spine xrays, ultrasound (FAST) and pelvis and limbs xrays.

Rehabilitation protocol was taught to 24 patients who were treated non operatively with DLSO brace and kept immobilised for 4-6 weeks and gradual mobilisation initiated with monthly follow up and strict neurological charting done. (fig 3)

16 patients were treated with operative intervention (fig 2) with short or long segmented fixation \& fusion with bone grafts. Follow up rehabilitation was similar as non operative patients except wound care and dressing with higher systemic followed by oral antibiotics regime. Patients with neurodeficit were trained and move around in wheel chair. Patients with complete paraplegia were kept over water bed and frequent log rolling advocated.

Patients neurological status and functional assessment were graded with Frankel score. kyphotic angle was measured in Preoperative and postoperative patients as well as patients with conservative management.

\section{Surgical technique}

All the patients were operated with posterior pedicle screwrod assembly (stainless steel or titanium).

Surgery was performed under general anaesthesia with patient in prone position. A posterior mid-line approach was used.

The subcutaneous tissues, erector spine muscle were infiltered with adrenalin solution subcutaneously to reduce the bleeding. The incision was deepened through the skin, subcutaneous tissue and dorsolumbar fascia to the tip of spinous process.

The paraspinal muscles where sub periosteally detached from the tip of the spinous process.

Entry points of the pedicle screws where made with the help of trochar. Pedicle was probed in all four quadrants to be sure that a solid tube of bone exists. Screw tap was introduced to 
cut the threads in the created tracts.

Rods of appropriate sizes were selected and contoured to maintained the normal lumbar lordosis. Screws of appropriate length were selected so that penetration into the vertebral body was approximately $80 \%$. (Fig 1)

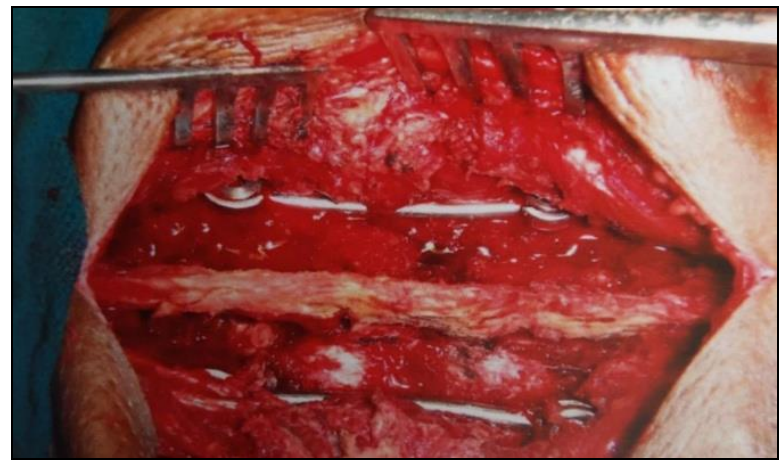

Fig 1: Fixation with rods

Reduction was achieved by distraction technique (ligamentotaxis).

Bone grafting was done after taking cancellous bone graft from the posterior superior iliac spine and wound was closed. Spinal cord was decompressed by laminectomy after insertion of pedicle screws and rods.

Thorough irrigation of the wound was done with antibiotic solution, closed suction drain was inserted and wound was closed in layers.

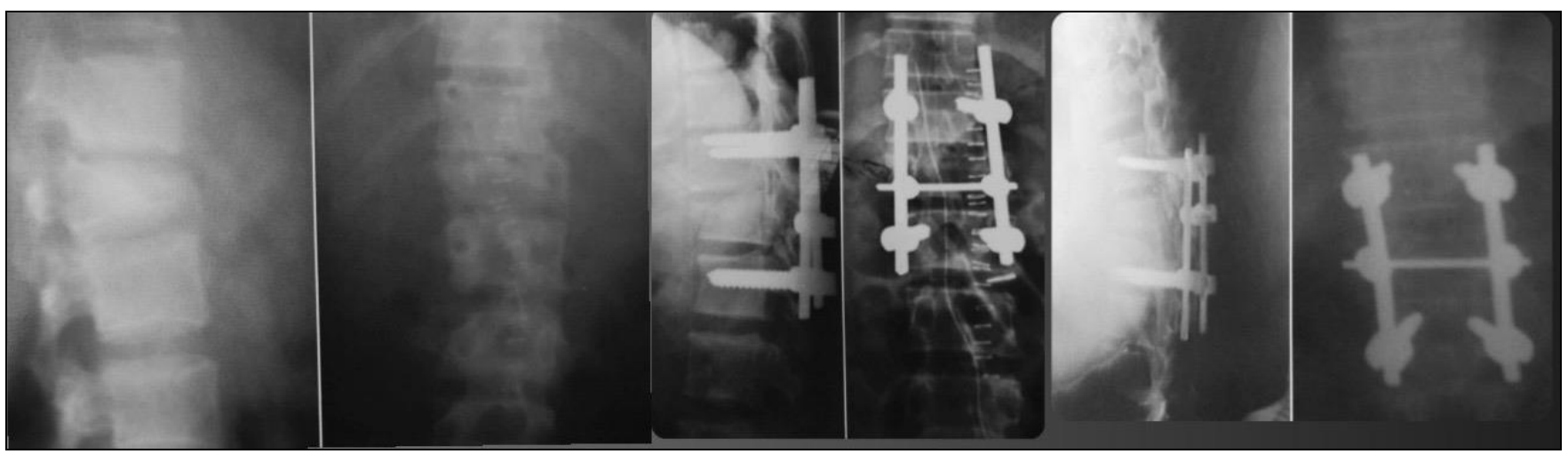

Fig 2: Showing preop, immediate post op and follow-up of operative patient

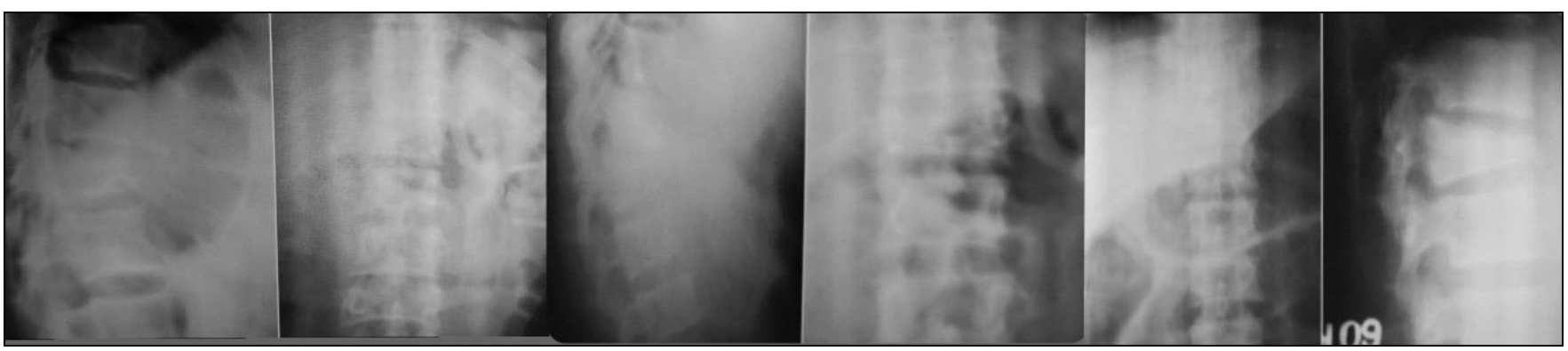

Fig 3: Showing immediate, post 1 month and 6 month followup of conservatively managed patient

\section{Results \& discussion}

40 patients with dorsolumbar spine injuries were treated at our institute, among them 6 patients (15\%) were 10-20 years of age, 14 patients $(35 \%)$ were between $20-30$ years of age while 11 patients $(28 \%)$ of patients were between $30-40$ years of age. 9 patients (22\%) were between 40-50 years of age group. 34 patients $(85 \%)$ were male while 6 patients $(15 \%)$ were female population in the study.

19 patients (47\%) had occupation before fall was daily wage labour work, 5 patients (13\%) were farmers, 4 patients $(10 \%)$ were housewives while 12 patients $(30 \%)$ had their own private office business. 28 patients $(70 \%)$ had fall from height while 3 patients $(7.5 \%)$ had fall of heavy objects. 8 patients had met with road traffic accidents.

Table 1: Classification of fractures

\begin{tabular}{|c|c|c|c|}
\hline S. No. & Type of fracture & No of patients & 24 \\
\hline 1 & Wedge Compression Fracture & 9 & 60 \\
\hline 2 & Burst Fracture & 3 & 22 \\
\hline 3 & Flexion Distraction Injuries & 4 & 10 \\
\hline 4 & Fracture Dislocation & 40 & 100 \\
\hline
\end{tabular}


24 patients $(60 \%)$ had wedge compression fracture at dorsolumbar region while 9 patients $(22 \%)$ had burst vertebral body fracture and remaining had flexion distraction injury (8\%) and fracture dislocation (10\%). 26 patients had L1 vertebra involved while 12 patients had D12 vertebral body injured. 18 patients $(45 \%)$ were in Frankel grade $\mathrm{E}$ while $25 \%$ of patients were in grade $\mathrm{A}$.

$31 \%$ (4 patients) had associated injury fracture radius ulna while $46 \%$ (6 patients) had fracture bimalleolar ankle and fracture calcaneus. 16 patients were selected for operative intervention while 24 patients were treated conservatively. $50 \%$ (8 patients) had interval between injury and surgery was 3-7 days while rest of the patients had 2 days to 21 days. 15 patients $(94 \%)$ were operated with monoblock mossmiami system of fixation and 1 patient was operated with stefee fixation system. 44\% (7 patients) who were operated with fixation system bone grafting was used. All patients were examined in follow up from 9 months to 24 months.

Table 2: Mode of Treatment

\begin{tabular}{|c|c|c|}
\hline & Operatively Treated & Non-Operatively Treated \\
\hline No of Patients & 16 & 24 \\
\hline Percentage & 40 & 60 \\
\hline
\end{tabular}

$12 \%$ (5 patients) had infection among operatively treated group which was serous in nature and mild in amount without systemic manifestations like fever or gross laboratory parameter abnormality while 3 patients had hardware failure in later period of followup.7 patients had bed sore which was superficial in nature without any form of discharge or deep infection. 3 patients treated conservatively had urinary infection while 2 patients had bed sore and 1 patient had deep vein thrombosis.

At follow up on regular check of neurological status, $50 \%$ of operatively treated patients were showing improvement. Remaining operative group patients had complete cord damage clinically as well as radiologically. Patients treated non operatively had no or occasional pain according to pain score.

10 patients had excellent score treated operatively while 12 patients had good score. 8 patients treated non operatively had good to fair score according to grading for neurological improvement by SRS(scoliosis research society) by Gertzbein.

\section{Conclusion}

A detailed history regarding mechanism and duration of injury is important along with thorough clinical and neurological examination. Associated injuries should always be noted thoroughly. We have found excellent results in non operative treatment in a stable spine with normal neurology as per Danish criteria. Rate of complications were less in nonoperative patients as compared to operated patients. So have concluded that surgical management might still be restricted in the patients with incomplete spinal cord injuries. Posterior screw stabilization gives rigid stabilization, good clearance of canal with satisfactory decompression of the spinal cord and allows early rehabilitation which shortens the hospital stay. Surgical intervention should be advocated in cases of unstable burst fractures of the dorsolumbar region, irrespective of status of neurological damage. The outcomes for patients treated with bone grafting do not differ in terms of complications like infection, implant failure, etc and rigid stable fixation with ideal pedicle screw placement is more important with patients with unstable fractures. Neurologically deficient unstable vertebral fracture patients should be operated rather than stabilizing the spine nonoperatively with early rehabilitation to prevent further kyphosis and to reduce pain due to spinal instability.

\section{References}

1. $\mathrm{Hu} \mathrm{R}$, Mustard CA, Burns C. Epidemiology of incident spinal fracture in a complete population. Spine. 1996; 15;21(4):492-9.
2. Denis, Francis. The three column spine and its significance in the classification of acute thoracolumbar spinal injuries. Spine. 1983; 8(8):817-31.

3. Ramani PS, Singhania BK, Murthy G. Combined anterior and posterior decompression and short segment fixation for unstable burst fractures in the dorso lumbar region. Neurology India. 2002; 1;50(3):272.

4. Dai LY. Principles of management of thoracolumbar fractures. Orthop Surg. 2012; 4:67-70.

5. Rajasekaran S. Thoracolumbar burst fractures without neurological deficit: the role for conservative treatment. European Spine Journal. 2010; 1;19(1):40-7.

6. Shimer $\mathrm{AL}, \mathrm{Su} \mathrm{BW}$. Operative versus nonoperative treatment of thoracolumbar burst fractures. In Seminars in Spine Surgery. WB Saunders. 2010; 22(1):38-43.

7. Denis F. The three column spine and its significance in the classification of acute thoracolumbar spinal injuries. Spine (Phila Pa 1976). 1983; 8:817-31.

8. Aebi M. Classification of thoracolumbar fractures and dislocations. Eur Spine J. 2010; 19Suppl1:S2-7.

9. McAfee PC, Yuan HA, Fredrickson BE, Lubicky JP. The value of computed tomography in thoracolumbar fractures. J Bone Joint Surg Am. 1983; 65(4):461-73.

10. McCormack T, Karaikovic E, Gaines RW. The load sharing classification of spine fractures. Spine. 1994; $1 ; 19(15): 1741-4$.

11. Lee JY, Vaccaro AR, Lim MR, Öner FC, Hulbert RJ, Hedlund $\mathrm{R}$ et al. Thoracolumbar injury classification and severity score: a new paradigm for the treatment of thoracolumbar spine trauma. Journal of Orthopaedic Science. 2005; 1;10(6):671.

12. Weinstein JN, Collalto PA, Lehmann TR. Thoracolumbar burst fractures treated conservatively: a long-term followup. Spine. 1988; 13(1):33-8.

13. Shen WJ, Liu TJ, Shen YS. Nonoperative treatment versus posterior fixation for thoracolumbar junction burst fractures without neurologic deficit. Spine. 2001; $1 ; 26(9): 1038-45$.

14. Chow GH, Nelson BJ, Gebhard JS, Brugman JL, Brown CW, Donaldson DH. Functional outcome of thoracolumbar burst fractures managed with hyperextension casting or bracing and early mobilization. Spine. 1996; 15;21(18):2170-5.

15. Benson DR, Burkus JK, Montesano PX, Sutherland TB, McLain RF. Unstable thoracolumbar and lumbar burst fractures treated with the AO fixateur interne. Clinical Spine Surgery. 1992; 1;5(3):335-43.

16. Shen WJ, Shen YS. Nonsurgical treatment of threecolumn thoracolumbar junction burst fractures without neurologic deficit. Spine. 1999; 15;24(4):412-5. 
17. McNamara MJ, Stephens GC, Spengler DM. Transpedicular short-segment fusions for treatment of lumbar burst fractures. Clinical Spine Surgery. 1992; 1;5(2):183-7.

18. Wood KB, Buttermann GR, Phukan R, Harrod CC, Mehbod A, Shannon B et al. Operative compared with nonoperative treatment of a thoracolumbar burst fracture without neurological deficit: A prospective randomized study with follow-up at sixteen to twenty-two years. J Bone Joint Surg Am. 2015; 97:3-9. 